\title{
Scheduling Smart Home Appliances Using Mixed Integer Linear Programming
}

\author{
Kin Cheong Sou, James Weimer, Henrik Sandberg, and Karl Henrik Johansson
}

\begin{abstract}
This paper considers the minimum electricity cost scheduling problem of smart home appliances. Operation characteristics, such as expected duration and peak power consumption of the smart appliances, can be adjusted through a power profile signal. The optimal power profile signal minimizes cost, while satisfying technical operation constraints and consumer preferences. Constraints such as enforcing uninterruptible and sequential operations are modeled in the proposed framework using mixed integer linear programming (MILP). Several realistic scenarios based on actual spot price are considered, and the numerical results provide insight into tariff design. Computational issues and extensions of the proposed scheduling framework are also discussed.
\end{abstract}

\section{INTRODUCTION}

Electricity consumption varies between different hours of the day, between days of the week, and between seasons of the year, where the highest power demand typically occurs when the outdoor temperature drops. In recent years, the power demand has reached new peak levels and created extra stress to balance demand and generation. Environmental and economical reasons will, in the near future, require distribution companies to consider more complex power balance scenarios based on the introduction of large scale renewable electricity generation, personal electrical vehicles (PEVs) and distributed electricity generation in residential areas. Intermittent renewable energy sources, such as wind, are dynamic by definition and will require additional balancing power to maintain quality of electrical supply to consumers. Additionally, an increasing number of PEVs will introduce high electricity consumption that is not always predictable. Both the wind power's dynamic contribution to electricity generation and the PEVs' random demand of electricity require a balancing force in the electricity grid.

Load balancing of urban electrical loads, such as residential/industrial electricity consumption, can be accomplished by minimizing the usage of non-renewable generation and scheduling controllable loads to times when renewable energy generation is high. Particular ways to engage the consumers in participating in load balancing is achieved through economic incentives such as time-varying electricity tariff (e.g. spot pricing [1]), or $\mathrm{CO}_{2}$ footprint for environmentally concerned consumers (e.g. the Stockholm Royal Seaport

The authors are with the ACCESS Linnaeus Center and the Automatic Control Lab, the School of Electrical Engineering, KTH Royal Institute of Technology, Sweden. \{sou, weimerj, hsan, kallej\}akth. se This work is supported by the Swedish Energy Agency, the Swedish Governmental Agency for Innovation Systems (VINNOVA), the Swedish Foundation for Strategic Research (SSF) and the Knut and Alice Wallenberg Foundation. project [2]). An illustration of spot price is shown in Fig. 1.

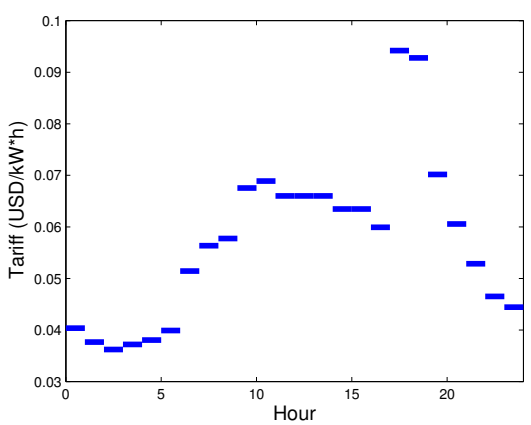

Fig. 1. Electricity tariff (spot price) for New York City on February 15th, 2011. Data taken from NYISO. The web address is www. nyiso.com.

References such as [3]-[5] have demonstrated the value of time-varying electricity tariff in the management of the power grid, especially in the reduction of peak power consumption; however, such load balancing is feasible only if the consumers are both able and willing to consider tariff information. For instance, it is unrealistic to expect most consumers to identify the most economical operation of their appliances in the presence of dynamic tariff prices and peak consumption penalties. Hence, an automatic decision support system is highly desirable, that either directly takes control of the appliance operation or provides simple advice to the consumers.

Prior works exist for load balancing from both industrial and residential consumers' perspective. For example, [6] describes the use of linear programming to schedule the electricity use of an industrial consumer for product manufacturing. Under various assumptions and model restrictions, [7] derives analytical formulas and scheduling strategies for an industrial consumer. A reinforcement learning based appliance scheduling framework for residential consumers is introduced in [8], assuming that both the tariff and the consumers' requests for appliances operation follow some Markov decision model.

In this paper, we also consider the problem of residential appliances scheduling; however, unlike [8], the proposed scheduling framework is deterministic and the cost calculation is based on the tariff, typically known 24 hours in advance. In addition, this work aims to model the decision problem as realistically as possible. Hence, simplified analytical and linear programming based analysis tools are not sufficient for the purpose of this work. Instead, we intention- 
ally explore the full modeling power of mixed integer linear programming (MILP). While the planning is 24 hours ahead, in practical household applications "real-time" adjustments are often required. Thus, this paper conducts a numerical study and demonstrates that in a typical scheduling scenario, prematurely terminating the MILP solving can result in very good suboptimal schedules. At the same time, the solve time can be reduced dramatically and is shown to be viable even for real-time household applications.

The remainder of this paper is organized as follows. Section II motivates and describes the appliance scheduling problem. Section III formulates the appliance scheduling problem into a MILP problem. Numerical studies using the MILP framework is provided in Section IV.

\section{Scheduling Home Appliances}

In this section, we provide a written description of the appliance scheduling problem which will be mathematically formulated as a MILP problem in the following section. In the proposed scheduling framework, an appliance operation process is divided into a set of sequential energy phases. An energy phase is an uninterruptible sub-task of the appliance operation which uses a pre-specified amount of electric energy. The energy phases are sequential since the next appliance sub-task cannot begin until the previous sub-task is completed (e.g. the washing machine agitator cannot start until the basin is filled with water).

In addition to being uninterruptible and having a specified energy usage, each energy phase may have additional manufacture-defined constraints. These constraints for a specific energy phase include bounding the instantaneous power consumed (corresponding to the maximum operating power and idle power) and achieving a maximum execution time. While all energy phases associated with a single appliance must be run sequentially, there can be delays between the energy phases so long as the energy phase ordering is preserved (e.g. the washing machine agitator must start within ten minutes of the basin being filled).

Besides the technical requirements for an energy phase specified by the manufacturer, there exist additional appliance-level operational constraints. For instance, a certain appliance cannot start before some other appliance finishes (e.g. washing machine and dryer). Moreover, for safety reason the total power assigned to all appliances at any moment cannot exceed a limit called peak signal. Finally, there might be user specified time preferences, requiring that certain appliances should be run within some particular time intervals (e.g. washing dishes with the dishwasher between $4 \mathrm{pm}$ and $6 \mathrm{pm})$.

To meet the appliance energy phase and operational constraints, the proposed appliance scheduling framework determines the power assignments, as functions of time over the execution period (e.g. a day), to all energy phases of all appliances. The time-dependent power assignments are called power profiles and each corresponds to an appliancespecific energy phase. The objective of the proposed scheduler is to find the least expensive set of power profiles, while satisfying the necessary operational constraints. To mathematically design the appliance scheduler, the following section introduces a MILP problem based on the appliance scheduling problem described in this section.

\section{MiXed Integer Linear Program Formulation}

The power profile scheduling decision problem in Section II can be modeled as a MILP problem (e.g. [9]). To define a MILP instance, a mathematical description of the problem setup, decision variables, cost function and constraints is provided below.

\section{A. Problem setup}

The appliances execution period is discretized into $m$ uniform time slots (e.g. 5 minutes per slot). The number of appliances considered for scheduling is denoted $N$, and the number of uninterruptible energy phases for each appliance is denoted $n_{i}$ for $i=1,2, \ldots, N$. Note that in this paper "appliance" and "energy phase" are abstractions. For instance, a single oven for lunch and dinner can be treated as two separate appliances.

\section{B. Decision variables}

The discretized power profiles are the output of the proposed scheduler and are denoted $p_{i j}^{k}$, corresponding to the energy assigned to energy phase $j$ of appliance $i$ during the whole period of time slot $k$. The typical unit for $p_{i j}^{k}$ is $\mathrm{kWh}$. The power profiles $p_{i j}^{k}$ are real (i.e., continuous) decision variables.

In addition to $p_{i j}^{k}$, auxiliary binary decision variables are required to indicate whether a particular energy phase is being processed or not. These binary decision variables are denoted $x_{i j}^{k} \in\{0,1\} \cdot x_{i j}^{k}=1$ if and only if for appliance $i$, energy phase $j$ is being processed during time slot $k$. The decision variables $x_{i j}^{k}$ are required, for instance, to model the energy phase sequential operation constraint described in Section II. In addition, two other sets of binary decision variables are needed to model the decision problem. One set is denoted as $s_{i j}^{k}$, with a value of one indicating that, in appliance $i$, energy phase $j$ is already finished by time slot $k$. The other set of auxiliary binary decision variables is denoted as $t_{i j}^{k}$. These decision variables are used to indicate whether at time slot $k$, appliance $i$ is making a transition between running phase $j-1$ to $j$. Hence, the index $j$ ranges from 2 to $n_{i}$ only (i.e., the number of energy phases in appliance $i$ minus one).

More explanation regarding the use of the auxiliary binary decision variables will be given in Section III-D.

\section{Cost function}

The objective of the proposed scheduler is to minimize the total electricity cost for operating the appliances. In this paper the cost calculation is based on a given 24-hour ahead electricity tariff (e.g. USD per $\mathrm{kWh}$ ). The tariff curve is piecewise constant, with possible jumps at the start of each hour. Fig. 1 shows an example of a typical tariff curve. 
Let $c^{k}$ denote the electricity tariff for time slot $k$. The total electricity cost for running all appliances is

$$
\sum_{k=1}^{m} c^{k}\left(\sum_{i=1}^{N} \sum_{j=1}^{n_{i}} p_{i j}^{k}\right)
$$

\section{Constraints}

To ease the description, the constraints are organized into two groups - energy constraints and timing constraints.

\section{1) Energy Constraints:}

Energy phase energy requirement: To make sure that the energy phases fulfill their energy requirements, the following constraint is imposed:

$$
\sum_{k=1}^{m} p_{i j}^{k}=E_{i j}, \quad \forall i, j
$$

where $E_{i j}$ is the energy requirements for energy phase $j$ in appliance $i$. These are technical specifications from the appliances.

Instantaneous energy phase power assignment bounds: To model whether an energy phase is being processed during time slot $k$, as well as the lower and upper limits of power assignment to the phase, the following constraint is imposed:

$$
\underline{P}_{i j}^{k} x_{i j}^{k} \leq p_{i j}^{k} \leq \bar{P}_{i j}^{k} x_{i j}^{k}, \quad \forall i, j, k
$$

where $\underline{P}_{i j}^{k}$ and $\bar{P}_{i j}^{k}$ are appliance specific data characterizing the lower and upper limits of power assignment to the energy phases. Note that if $x_{i j}^{k}=0$, then the inequalities above collapse to a single condition $p_{i j}^{k}=0$.

Power safety: The power safety constraint (i.e., upper limit of the total energy assigned in any time slot) can be modeled as

$$
\sum_{i=1}^{N} \sum_{j=1}^{n_{i}} p_{i j}^{k} \leq \text { PEAK }^{k}, \quad \forall k
$$

where $\mathrm{PEAK}^{k}$ is the "peak signal" (i.e., total slot energy upper bound) at time slot $k$. The peak signal is provided by the external power grid operator, which can be a demand response signal.

\section{2) Timing Constraints:}

Energy phase process time limits: To model the limits on energy phase process time, the following constraint is enforced:

$$
\underline{T}_{i j} \leq \sum_{k=1}^{m} x_{i j}^{k} \leq \bar{T}_{i j}, \quad \forall i, j
$$

where $\underline{T}_{i j}$ and $\bar{T}_{i j}$ are the lower and upper limits of the number of time slots for energy phase $j$ in appliance $i$ to be processed.

Uninterruptible operation: An energy phase being uninterruptible means that it cannot be resumed. This can be modeled by the constraint that, for all $i$ and $j, x_{i j}^{k}=0$ if there exists an earlier time slot $\tilde{k}<k$ such that $x_{i j}^{\tilde{k}}=1$ and $x_{i j}^{\tilde{k}+1}=0$. An alternative constraint can be imposed with the aid of the auxiliary decision variables $s_{i j}^{k}$ introduced in Section III-B:

$$
\begin{array}{ll}
x_{i j}^{k} \leq 1-s_{i j}^{k} & \forall i, j, k \\
x_{i j}^{k-1}-x_{i j}^{k} \leq s_{i j}^{k} & \forall i, j, \forall k=2,3, \ldots, m \\
s_{i j}^{k-1} \leq s_{i j}^{k} & \forall i, j, \forall k=2,3, \ldots, m
\end{array}
$$

In constraint (6a), if $s_{i j}^{k}=1$, then during time slot $k$ energy phase $j$ in appliance $i$ is already finished. Hence, the corresponding $x_{i j}^{k}$ must be 0 . The condition triggering $s_{i j}^{k}=1$ is that $x_{i j}^{k}$ switch from 1 to 0 (i.e., the phase is just finished). This is the situation in (6b). Then $s_{i j}^{k}$ should remain unity, as (6c) imposes.

Sequential Processing: Sequential processing of the energy phases of an appliance means that an energy phase cannot be processed unless its preceding phases have finished. This condition can be conveniently described using the auxiliary decision variables $s_{i j}^{k}$ as follows:

$$
x_{i j}^{k} \leq s_{i(j-1)}^{k}, \quad \forall i, k, \forall j=2,3, \ldots, n_{i}
$$

Similarly, to model the sequential operation between appliances, a constraint similar to above can be imposed:

$$
x_{i 1}^{k} \leq s_{\tilde{i n}_{\tilde{i}}}^{k}, \quad \forall k
$$

with $\tilde{i}$ being the index of the appliance which must be finished before $i$ can start. In above, $s_{\tilde{i} \tilde{i}_{\tilde{i}}}^{k}$ pertains to appliance $\tilde{i}$, energy phase $n_{\tilde{i}}$ (i.e., the last phase of appliance $\tilde{i}$ ) and time slot $k$.

Between-phase delay: To count the number of time slots spent between the energy phases in an appliance, the auxiliary 0-1 decision variables $t_{i j}^{k}$ defined in Section III-B can be utilized. During any time slot $k, t_{i j}^{k}=1$ if and only if that appliance $i$ has finished processing energy phase $j-1$, and it is waiting to process the phase $j$ (i.e., phase $j$ is not being processed or finished). The corresponding constraint is

$$
t_{i j}^{k}=s_{i(j-1)}^{k}-\left(x_{i j}^{k}+s_{i j}^{k}\right), \quad \forall i, k, \forall j=2,3, \ldots, n_{i}
$$

Note that $x_{i j}^{k}+s_{i j}^{k} \leq 1$ because an energy phase cannot simultaneously be processed and finished (cf. (6a)). Hence, the equality in (9) is valid. With $t_{i j}^{k}$ defined, the constraint enforcing the lower and upper limits of the number of transition time slots (i.e., delay between energy phases) can be written as

$$
\underline{D}_{i j} \leq \sum_{k=1}^{m} t_{i j}^{k} \leq \bar{D}_{i j}, \quad \forall i, \forall j=2,3, \ldots, n_{i}
$$

In above, $\underline{D}_{i j}$ and $\bar{D}_{i j}$ are appliance technical specifications describing the between-phase delay bounds (lower and upper, respectively) in the number of time slots.

User time preference: The household user can set up the time preference constraints, specifying the time interval a particular appliance must be finished within. Alternatively, this means that the appliances cannot be run outside of the time preference interval. The constraints are written as

$$
x_{i j}^{k} \leq \mathrm{TP}_{i}^{k}, \quad \forall i, j, k
$$


where $\mathrm{TP}_{i}^{k}$ characterizes the time preference interval. That is, $\mathrm{TP}_{i}^{k}=0$ if and only if none of the energy phases of appliance $i$ can be processed during time slot $k$.

\section{E. MILP formulation}

To sum up, the proposed minimum electricity cost appliance scheduling problem can be summarized into

$$
\begin{array}{cl}
\underset{p, x, s, t}{\operatorname{minimize}} & \text { cost function (1) } \\
\text { subject to } & \text { constraints }(2)-(11) \\
& p_{i j}^{k} \in \mathbb{R}, \quad \forall i, j, k \\
& x_{i j}^{k} \in\{0,1\}, \quad \forall i, j, k \\
& s_{i j}^{k} \in\{0,1\}, \quad \forall i, j, k \\
& t_{i j}^{k} \in\{0,1\}, \quad \forall i, k \forall j=2, \ldots, n_{i}
\end{array}
$$

The MILP problem such as (12) is a classical optimization paradigm. It can be solved using algorithms such as branchand-bound and cutting-plane method (e.g. [9], Chapter 11). Commercial (and academic) implementations of these algorithms are available (e.g. CPLEX and Gurobi).

\section{Numerical Studies}

All experiments in this paper are performed on a laptop with an Intel Core i5 2.53GHz CPU and 4GB of memory.

\section{A. Two case studies based on tariffs in Sweden and NYC}

In this experiment, two instances of the MILP scheduling problem in (12) are solved using CPLEX (using the YALMIP MATLAB interface [10]). In the first instance, the electricity tariff (i.e., $c^{k}$ in (1)) is taken to be the spot price on Feb 15th, 2011 in Sweden. Fig 2 shows the Swedish tariff. On

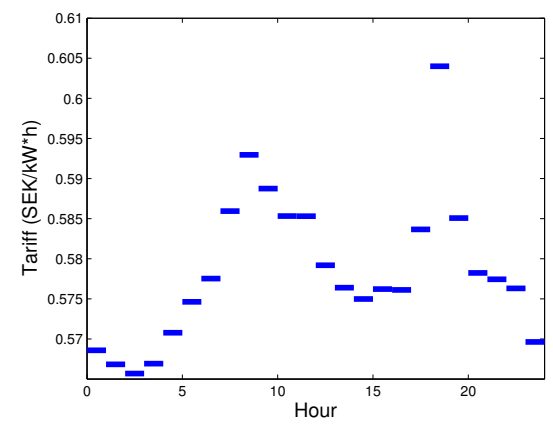

Fig. 2. Electricity tariff (spot price) for Sweden on February 15th, 2011. Data are taken from Nord Pool Spot. The web address is www. nordpoolspot. com.

the other hand, the tariff in the second instance is the spot price of New York City on Feb 15th, 2011 in Fig. 1.

The (planned) execution period is from 9am to the end of the day. The length of the time slots is 5 minutes. There are three controllable smart appliances including a dishwasher, a washing machine and a dryer. The household user imposes the following time preference (cf. (11) in Section III-D). The dishwasher is run between the beginning of $7 \mathrm{pm}$ and the end of the day. The washing machine and dryer can be run anytime between the beginning of 9am and the end of $11 \mathrm{pm}$. However, the washing machine phases must be finished before the dryer can start. The above specifies the constraints in (8) and (11). The values of the technical specifications in remaining constraints can be found in the Appendix. Finally, the peak signal in (4) is assumed to be constant, and is always equal to $5500 \mathrm{Wh}$.

After solving the first instance (with $c^{k}$ being the Swedish spot price), the sum of energy assigned to all appliances in each time slot, as well as the electricity tariff, are plotted in Fig. 3, which verifies that the proposed scheduler assigns electricity usage when it is cheap.

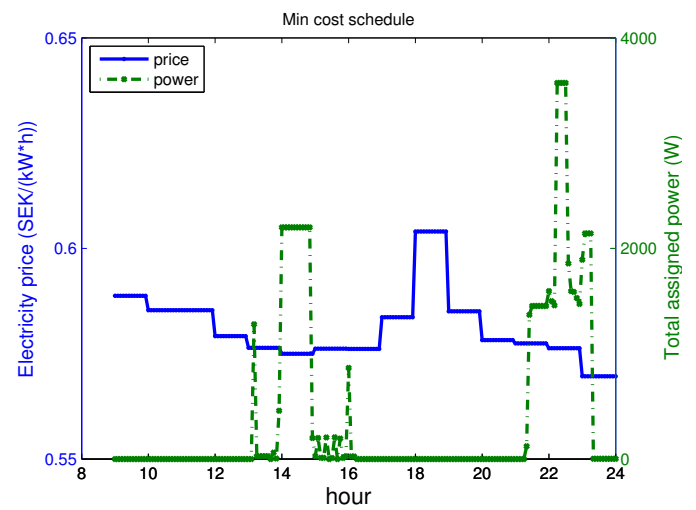

Fig. 3. Total energy assignment (for each time slot) and the electricity tariff, in the Sweden case. The minimum cost schedule assigns energy only when the tariff is low, subject to the constraints from (2) through (4).

The analogous result for solving the instance with the NYC tariff are shown in Fig. 4. Fig. 5 also shows the detailed

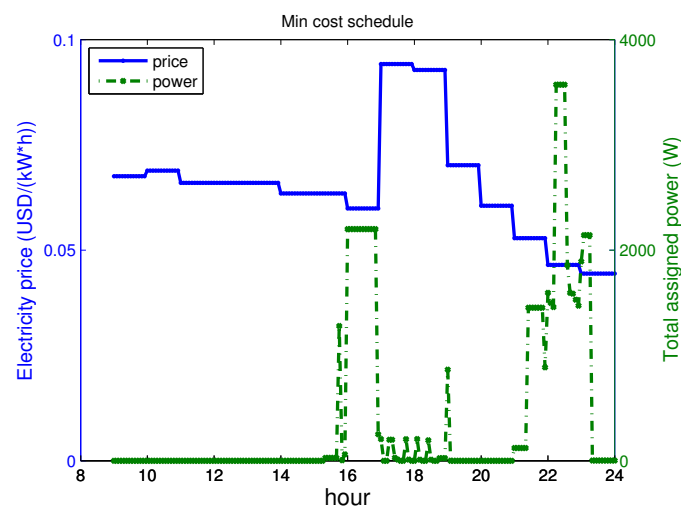

Fig. 4. Total energy assignment (for each time slot) and the electricity tariff, in the NYC case. The minimum cost schedule assigns energy only when the tariff is low, subject to the constraints from (2) through (4).

power profiles achieving the minimum cost in the NYC case.

The optimal electricity cost in the Sweden case is 3.528 SEK (about 105 SEK a month if the schedule is applied every day). On the other hand, the optimal electricity cost in the NYC case is 0.3256 USD (about 9.767 USD a month if the same schedule is applied every day). To understand how much cost saving the proposed scheduling framework can achieve, the optimal scheduling problem in (12) can be turned into the worst case scheduling problem by changing the cost function in (12) from minimization to maximization. 

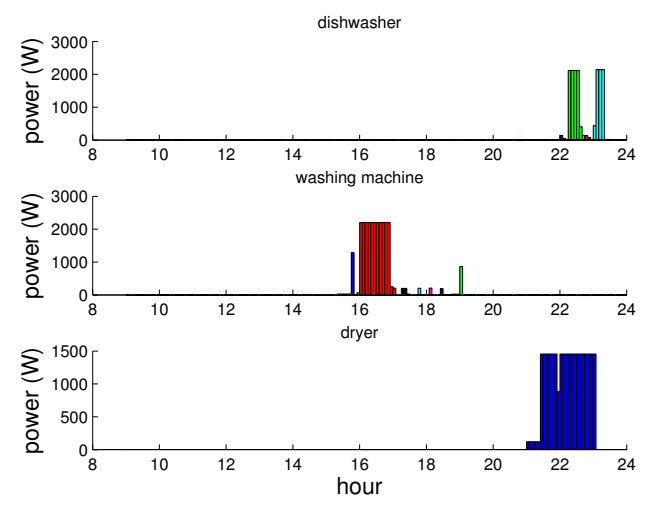

Fig. 5. The minimum cost power profiles for the appliances in the NYC tariff case. Different energy phases are painted with different colors.

For the Sweden case, the worst cost is 3.616 SEK (about $2.5 \%$ more than the optimal cost). The saving is quite insignificant in this case. On the other hand, for the NYC case, the worst cost is 0.4781 USD (about $47 \%$ more than the optimal cost). The case studies here confirms the intuition that tariff fluctuation needs to be large enough to motivate changes of power consumption behavior. In the NYC case, the ratio of the maximum and minimum tariff is 2.601. On the other hand, in the Swedish case the ratio of the maximum and minimum tariff is only 1.068 . With the maximum saving of only $2.5 \%$, there is no motivation for the consumers to change their behaviors.

\section{B. Computation time experiment}

For real-time household application, the solving of the scheduling problem in (12) cannot be too time-consuming. In (12), the tuning parameter which is responsible for the tradeoff between computation time and model fidelity is the length of the time slot. Table I shows the statistics of solving the NYC instance with three different values of time slot lengths. Table I suggests that while the time

TABLE I

SOLVING THE NYC INSTANCE WITH DIFFERENT TIME SLOT LENGTHS

\begin{tabular}{|c|c|c|c|c|}
\hline time slot length & min cost & max cost & max saving & solve time \\
\hline $3 \mathrm{~min}$ & $\$ 0.3257$ & $\$ 0.4801$ & $47.43 \%$ & $860 \mathrm{sec}$ \\
\hline $5 \mathrm{~min}$ & $\$ 0.3256$ & $\$ 0.4781$ & $46.84 \%$ & $83.6 \mathrm{sec}$ \\
\hline $10 \mathrm{~min}$ & $\$ 0.3251$ & $\$ 0.4780$ & $47.05 \%$ & $15.4 \mathrm{sec}$ \\
\hline
\end{tabular}

slot length has a significant impact on computation time, its effect on the optimal cost is not very obvious. This justifies the use of lower fidelity optimization models, so long as the temporal constraints (e.g. process time bounds) are reasonably captured.

The MILP solver CPLEX has the ability to prematurely terminates the optimal solution search. In particular, CPLEX can stop as soon as it finds a feasible solution of (12). Also, CPLEX allows the user to specify a time limit upon which the solving is terminated, irrespective of whether a feasible/optimal solution has been found or not. In the following experiment, CPLEX is used to first find a feasible solution (this is the minimum requirement of using CPLEX in the appliance scheduling problem). Then the feasible solution is used as an initial guess for the next CPLEX runs, with variable solve time limits. The costs of these runs are then compared against the optimal cost obtained by a single CPLEX solve (i.e., the second and third rows in Table I). The results of the experiment with the NYC case with the time slot lengths being 10 and 5 minutes are shown in Table II.

TABLE II

RELATIVE ERROR VS CPLEX ALLOWED RUNTIME. NYC CASE WITH DIFFERENT TIME SLOT LENGTHS $d$ (IN MINUTES). IN THE $d=10$ AND $d=5$ CASES THE FIRST FEASIBLE SOLUTIONS ARE OBTAINED, RESPECTIVELY, AFTER 1.19 AND 6.94 SECONDS

\begin{tabular}{|c|l|c|c|c|c|c|c|}
\hline \multirow{2}{*}{$d=10$} & runtime (s) & 1.19 & 2.42 & 4.55 & 6.41 & 11.4 & 14.4 \\
\cline { 2 - 8 } & rel. err. \% & 0.46 & 0.46 & 0.058 & 0.051 & 0.051 & $\approx 0$ \\
\hline \hline \multirow{2}{*}{$d=5$} & runtime (s) & 6.94 & 12.2 & 17.2 & 27.2 & 67.2 & 87.2 \\
\cline { 2 - 8 } & rel. err. \% & 0.34 & 0.34 & 0.34 & 0.20 & 0.20 & $\approx 0$ \\
\hline
\end{tabular}

Table II suggests that the suboptimal power profiles returned by prematurely terminating CPLEX are acceptable substitutes for the true optimal profiles which are much more time consuming to find. In particular, in the 10-minute time slot case the 1-second feasible solution incurs no more than $0.5 \%$ of relative error (w.r.t. the true optimal cost 0.3251 in Table I). In the 5-minute time slot case the 7-second feasible solution incurs no more than $0.35 \%$ of relative error. The above experiment demonstrates that it is promising to apply the proposed framework in the real-time household appliances scheduling scenario.

\section{Scalability Test}

To test the limit of computation time and memory requirements for solving (12), hypothesis scenarios are considered with increasing number of appliances (each appliance has 6 energy phases). In these scenarios, the energy and timing constraints in Section III-D are randomly specified. The NYC tariff is chosen, and the length of the time slots is 10 minutes.

The random scenarios are solved using (a) CPLEX to optimality, (b) CPLEX for the first feasible solution as in Section IV-B, (c) a simple strategy to run the appliances as soon as possible (ASAP), provided that the constraints are satisfied, and (d) the analogous as late as possible (ALAP) strategy. CPLEX runs out of memory for solving the scenario with 10 appliances. The method for finding the first feasible solution fails with 20 appliances. This verifies the intuition that (12) does not admit scalable solution algorithms, and it should be restricted to the case of a single household with a few appliances (e.g. less than five). Figure 6 shows the experiment results for the scenarios with less than 9 appliances. The solve time to optimality increases rapidly as the number of appliances increases. On the other hand, for the first feasible solution only the solve time remains reasonable (about $40 \mathrm{sec}$ for 9 appliances) with good approximation quality (less than $5 \%$ of relative increase in electricity cost). Note also that the ASAP strategy fares well, but the ALAP one is inaccurate. Since the accuracy of the ASAP and ALAP schemes depends sensitively on the tariff, the validity of these schemes are questionable. 


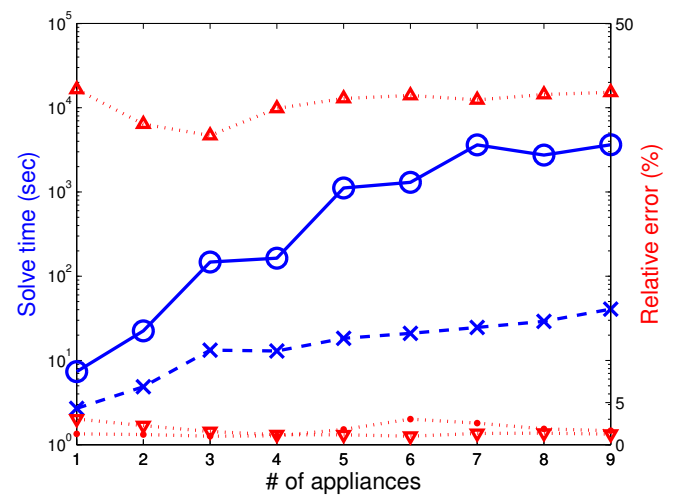

Fig. 6. Computation cost and relative error for solving randomly generated scenarios with different number of appliances. Blue solid line with circles: solve time for CPLEX optimal; Blue dashed line with " $x$ ": solve time for CPLEX first feasible solution; Red dotted line with dots: CPLEX first feasible solution relative error; Red dotted line with downward pointing triangles: ASAP relative error; Red dotted line with upward pointing triangles: ALAP relative error.

\section{CONCLUSION}

In this paper a MILP based smart appliance scheduling framework is proposed, capturing all relevant appliance operations. With appropriately defined tariff (e.g. the NYC case), the proposed framework can result in a scheduling achieving about $47 \%$ of maximum cost saving. In addition, it is demonstrated that good quality approximate solutions can be obtained in a reasonable amount of computation time (e.g. in about 1 second an approximate solution with relative error less than $0.5 \%$ can be obtained). Finally, the proposed framework can be extended to incorporate renewable energies, battery and the multi-objective optimization with respect to energy consumption and $\mathrm{CO}_{2}$ footprint.

\section{ACKNOWLEDGEMENTS}

The authors are grateful to the Stockholm Royal Seaport project collaborators including Electrolux and ABB.

\section{REFERENCES}

[1] F. Schweppe, M. Caramanis, R. Tabors, and R. Bohn, Spot pricing of electricity. Boston, MA, USA: Kluwer Academic Publishers, 1988.

[2] "Stockholm royal seaport project," www.stockholmroyalseaport.com.

[3] A. Sanghvi, "Flexible strategies for load/demand management using dynamic pricing," Power Systems, IEEE Transactions on, vol. 4, no. 1, pp. $83-93$, Feb. 1989.

[4] J. Pyrko, "Load demand pricing - case studies in residential buildings," in International Energy Efficiency in Domestic Appliances and Lighting Conference, 2006.

[5] P. Fritz and E. Jörgensen and S. Lindskoug, "Elforsk technical report 09:70," Elforsk, Tech. Rep., 2009, Report written in Swedish with English summary. Available online from http://www.elforsk. se/Rapporter/?rid=09_70.

[6] B. Daryanian, R. Bohn, and R. Tabors, "Optimal demand-side response to electricity spot prices for storage-type customers," Power Systems, IEEE Transactions on, vol. 4, no. 3, pp. 897 -903, Aug. 1989.

[7] J. Roos and I. Lane, "Industrial power demand response analysis for one-part real-time pricing," Power Systems, IEEE Transactions on, vol. 13, no. 1, pp. 159 -164, Feb. 1998.

[8] D. ONeill, M. Levorato, A. Goldsmith, and U. Mitra, "Residential demand response using reinforcement learning," in IEEE SmartGridComm, 2010.

[9] J. Tsitsiklis and D. Bertsimas, Introduction to Linear Optimization. Athena Scientific, 1997.
[10] J. Löfberg, "Yalmip : A toolbox for modeling and optimization in MATLAB," in Proceedings of the CACSD Conference, Taipei, Taiwan, 2004. [Online]. Available: http://users.isy.liu.se/johanl/yalmip

[11] A. Rugo, "Power profiles for smart appliances," Private communication, ELECTROLUX ITALIA S.P.A.

\section{APPENDIX}

\section{A. Technical specifications of the smart appliances}

The number of energy phases in the dishwasher, washing machine and dryer processes are, respectively, 6,8 and 1 . The data of the scheduling problem are derived from the technical specifications of the three appliances, which are listed in Table III, Table IV and Table V respectively.

TABLE III

DISHWASHER TECHNICAL SPECIFICATIONS [11]

\begin{tabular}{|c|c|c|c|c|}
\hline Energy phase & $\begin{array}{c}\text { Energy } \\
(\mathrm{Wh})\end{array}$ & $\begin{array}{c}\text { Min power } \\
(\mathrm{W})\end{array}$ & $\begin{array}{c}\text { Max power } \\
(\mathrm{W})\end{array}$ & $\begin{array}{c}\text { Nominal op. } \\
\text { time (min) }\end{array}$ \\
\hline pre-wash & 16.0 & 6.47 & 140 & 14.9 \\
\hline wash & 751.2 & 140.26 & 2117.8 & 32.1 \\
\hline 1st rinse & 17.3 & 10.28 & 132.4 & 10.1 \\
\hline drain & 1.6 & 2.26 & 136.2 & 4.3 \\
\hline 2nd rinse & 572.3 & 187.3 & 2143 & 18.3 \\
\hline drain \& dry & 1.7 & 0.2 & 2.3 & 52.4 \\
\hline
\end{tabular}

TABLE IV

WASHING MACHINE TECHNICAL SPECIFICATIONS [11]

\begin{tabular}{|c|c|c|c|c|}
\hline Energy phase & $\begin{array}{c}\text { Energy } \\
(\mathrm{Wh})\end{array}$ & $\begin{array}{c}\text { Min power } \\
(\mathrm{W})\end{array}$ & $\begin{array}{c}\text { Max power } \\
(\mathrm{W})\end{array}$ & $\begin{array}{c}\text { Nominal op. } \\
\text { time (min) }\end{array}$ \\
\hline movement & 118 & 27.231 & 2100 & 26 \\
\hline pre-heating & 5.5 & 5 & 300 & 6.6 \\
\hline heating & 2054.9 & 206.523 & 2200 & 59.7 \\
\hline maintenance & 36.6 & 11.035 & 200 & 19.9 \\
\hline cooling & 18 & 10.8 & 500 & 10 \\
\hline 1st rinse & 18 & 10.385 & 700 & 10.4 \\
\hline 2nd rinse & 17 & 9.903 & 700 & 10.3 \\
\hline 3rd rinse & 78 & 23.636 & 1170 & 19.8 \\
\hline
\end{tabular}

TABLE V

DRYER TECHNICAL SPECIFICATIONS [11]

\begin{tabular}{|c|c|c|c|c|}
\hline Energy phase & $\begin{array}{c}\text { Energy } \\
(\mathrm{Wh})\end{array}$ & $\begin{array}{c}\text { Min power } \\
(\mathrm{W})\end{array}$ & $\begin{array}{c}\text { Max power } \\
\text { (W) }\end{array}$ & $\begin{array}{c}\text { Nominal op. } \\
\text { time (min) }\end{array}$ \\
\hline drying & 2426.3 & 120.51 & 1454 & 120.8 \\
\hline
\end{tabular}

In terms of the optimization problem in (12), the energy requirements $E_{i j}$ are listed in the "Energy" column in the tables. The lower and upper limits for energy assignment in each time slot, $\underline{P}_{i j}^{k}$ and $\bar{P}_{i j}^{k}$ in (3), are listed in the "Min power" and "Max power" columns in the tables, respectively. The last column in the above tables list the nominal operation time of all phases. In this paper, it is assumed that the operation time of the energy phases can be between $80 \%$ and $120 \%$ of the nominal time. The time slot limits $\underline{T}_{i j}$ and $\bar{T}_{i j}$ in (5) are obtained by dividing the operation time limits (in minutes) by the time slot length (i.e., 5 minutes), and rounding is performed where necessary. Finally, the lower limit for between-phase delay $\underline{D}_{i j}$ in (10) for all phases is assumed to be zero, while the upper limits $\bar{D}_{i j}$ are 5, 10 and 0 minutes for the energy phases in the dishwasher, washing machine and dryer respectively. 